\title{
Monitoring, analysis and post-casting of the Earth's particle radiation environment during February 14-March 5, 2014
}

\author{
Vladimir Kalegaev ${ }^{1, *}$, Mikhail Panasyuk ${ }^{1}$, Irina Myagkova ${ }^{1}$, Yulia Shugay ${ }^{1}$, Natalia Vlasova ${ }^{1}$, \\ Wera Barinova $^{1}$, Evgenia Beresneva ${ }^{1}$, Sergey Bobrovnikov ${ }^{1}$, Valery Eremeev ${ }^{1}$, Sergey Dolenko ${ }^{1}$, \\ Ilya Nazarkov ${ }^{1}$, Minh Nguyen ${ }^{1}$, and Arnaud Prost ${ }^{2}$ \\ ${ }^{1}$ Skobeltsyn Institute of Nuclear Physics of Lomonosov Moscow State University, 119991 Moscow, Russia
2 ISAE-SUPAERO, 31400 Toulouse, France
}

Received 13 February 2018 / Accepted 15 July 2019

\begin{abstract}
Internet-based system of Space Monitoring Data Center (SMDC) of Skobeltsyn Institute of Nuclear Physics of Moscow State University (SINP MSU) has been developed to predict and analyze radiation conditions in near-Earth space. This system contains satellite measurement databases and operational models and devoted to collect, store and process space weather monitoring data in the near real-time. SMDC operational services acquire data from ACE, SDO, GOES, Electro-L, Meteor-M satellites and use them for forecasting, now-casting and post-casting of space weather factors. This paper is intended to give overview of operational services of SMDC Internet-based system and demonstrate their possibilities and limitations to analyze space weather phenomena and predict radiation and geomagnetic conditions in the near-Earth space during February 14-March 5, 2014. This prolonged period of high level solar and geomagnetic activity demonstrates various manifestations of the space weather: solar proton events, geomagnetic storms and outer radiation belt (RB) dynamics. Solar sources of interplanetary space disturbances and their influence on geomagnetic and radiation state of the Earth's magnetosphere were described using output coming from SMDC' Web-based applications. Validation of SMDC's operational models was performed based on the quality of description of the physical conditions in near-Earth space during space weather events observed from February 14 to March 5, 2014. The advantages and disadvantages of SMDC operational services are illustrated and discussed based on comparison with data obtained from satellites.
\end{abstract}

Keywords: space weather / solar wind / magnetosphere / radiation belts / data mining

\section{Introduction}

Half a century after the beginning of the space age, radiation safety of space missions is becoming more and more critical. Physical (mainly, particle radiation) conditions in space controlled by solar activity (space weather) influence satellite operations and may provoke failures in electronic parts. With the development and increasing complexity of space technology humanity has become much more dependent on the processes that occur in near-Earth space (Albertson et al., 1973; Lanzerotti et al., 1999). Due to enhanced role of space-based technological systems (navigation, communication, etc.) in modern epoch, the ability to quickly assess and predict these processes is of the key importance for successful space activity (Baker et al., 2004). Operational control of the state of near-Earth's space and

*Corresponding author: klg@decl.sinp.msu.ru continuous monitoring of the key parameters of space environment is strongly needed to mitigate space weather risks. With an increasing number of space missions, there is the growing problem of operational receipt, storage and real time processing of the data from space measurements. These data as well as modern computing and communication tools however provide new opportunities for reliable analysis and forecasting of space environment conditions, based on operational applications developed during the last decade in world space weather centers.

Space weather, as an applied branch of space physics, is a strategically important field of science, especially for the leading space powers which have already a significant commercial and industrial presence in space (Schrijver et al., 2015). One of the most important factors of space weather is radiation. Actually, this phenomenon can have electromagnetic or corpuscular origin but in this paper we will consider only particle radiation. 
The Sun, directly (Solar Energetic Particles [SEPs] penetration into the magnetosphere) or indirectly (outer electron radiation belt (RB) variations during geomagnetic disturbances), influences the Earth's radiation environment. Monitoring of the radiation conditions in space gives information on particle fluxes at given orbits. On the other side, physical models of the space environment allow to reconstruct physical conditions in different space domains, based on measurements taken from a few spacecraft. In that regard, we need to continue the experimental and theoretical study of solar-terrestrial relationships and their possible impact on technological systems to get more measurement data and to develop more reliable models. Such studies are strongly needed to ensure the safety of the crew and passengers of aircraft on polar routes, and manned spaceflights, as well as to prevent failures of electronic equipment during the increase of fluxes of SEP.

The ultimate goal of research in this field is to create a complex set of coupled operational models "from Sun to Earth" (Schrijver et al., 2015) that allow to predict the radiation risk for spacecraft at different orbits, and also to estimate the occurrence risk of technological disasters due to magnetic storms and variations in charged particle fluxes. The solution of this problem is to collect all relevant space-related data sets from multiple satellites of various purposes and capabilities as well as from ground stations, and develop the operational services that use space weather models and collected data to provide forecasts. This approach becomes especially important in the modern epoch, since excellent technical capabilities exist to transfer data from satellite to Earth, as well as to process and visualize this data almost immediately.

A lot of space weather centers around the world provide realtime information on space conditions based on monitoring data and modeling: Space Weather Prediction Center (SWPC/ NOAA) (http://www.swpc.noaa.gov), iNTEGRATED Space Weather Analysis System (iSWA) (https://ccmc.gsfc.nasa.gov/ iswa/), European Space Situation Awareness (SSA) Space Weather system (http://swe.ssa.esa.int), as well as national and university centers. The main monitoring space missions provide real-time data for analysis and forecasting of space weather conditions based on operational models. The monitoring data contains usually measurements from geostationary satellites (NOAA/GOES), spacecraft in solar wind (ACE, WIND, DSCOVR), solar imagers (SDO, SOHO, STEREO), on-ground measurements from magnetic stations, ionospheric radars etc. Continuous satellite monitoring is the main part of space weather systems (Cheng et al., 2006; Schrijver et al., 2015; Granja et al., 2016; Kraft et al., 2019). Space weather centers collect the measurements and provide access to satellite data, and forecasting models online through the websites. Each set of data sources and models is unique in its own way, and usually represents some part of the whole picture of space weather.

SINP MSU has a strong expertise of particle detectors development. During a long time (starting from second Sputnik) it was responsible for radiation instrumentation onboard most of the satellites created in Soviet Union and in Russia. Space Monitoring Data Center (SMDC) of SINP MSU acquires and collects data from Russian satellites equipped by detectors manufactured at SINP MSU. Two web-sites (http://smdc.sinp.msu.ru) and the modern SMDC space weather site (http://swx.sinp.msu.ru, English version http://swx.sinp.msu.ru/index.php?lang=en) provide access to data on energetic particle fluxes obtained from Russian scientific missions (CORONAS series, Tatyana, Tatyana-2, Vernov, ...) as well as from Russian weather satellites (Electro-L and Meteor-M series). These measurements complement the data from GOES, ACE, DSCOVR, SDO and some other monitoring satellites as well as data from on-ground facilities that usually are used for space weather issues. Space Weather web-site (http://swx.sinp.msu.ru/) gives advanced possibilities for various datasets visualization and cross-comparison. Graphical applications give opportunity to analyze simultaneously data from different sources.

SMDC Space Weather web-site also provides access to operational and forecasting models of SMDC as web-based applications:

- the solar wind forecast at L1 point. In this application, the velocities of recurrent High-Speed Streams (HSS) are predicted for about of 3-day period based on the coronal hole's parameters;

- forecast of the solar wind parameters and Interplanetary Magnetic Field (IMF) at the Earth's orbit; plasma and magnetic field measurements at L1 point are used as input for simple propagation model;

- Dst index forecast in term of the perceptron type of artificial neural networks (ANNs) depending on solar wind measurements at L1 point;

- forecast of the fluxes of outer RB relativistic electrons with the energy greater than $2 \mathrm{MeV}$ based on perceptron type of ANNs.

All forecasts use the measurements collected in SMDC integrated database. Data obtained from GOES, ACE, DSCOVR, Electro-L1, Electro-L2, Meteor-M1, Meteor-M2 spacecraft as well as images from the SDO satellite are used as inputs for various SMDC operational services. Joint analysis of data coming from multi-satellite measurements as well as from operational models allows to carry out near real-time report on the state of the Earth's environment. One of the main scopes of SMDC is to provide to satellite operators in Russia the reliable information on radiation conditions in space to ensure the radiation safety of Russian spacecraft.

In this paper, we present the data that can be obtained and analyzed in the framework of SMDC Space Weather system during February 14-March 5, 2014 period when several space weather events took place. The detailed study of the monitoring data obtained during prolonged period with events of different nature gives good opportunity to reveal strong and weak aspects and demonstrate the advantages and disadvantages of SMDC Space Weather services. Overview of SMDC data sources and operational services that can be accessed by Space Weather Internet resource http://swx.sinp.msu.ru is presented in Section 2. This is followed by Section 3 where we describe the overall space weather conditions during prolonged period of solar and geomagnetic activity on February 14-March 5, 2014. Unlike the usual case studies devoted to analysis of the single specific event we take the long enough period containing a rich set of solar, interplanetary medium' and magnetospheric phenomena.

Implementations of operational models in the SMDC Space Weather system as well as SMDC data services give 


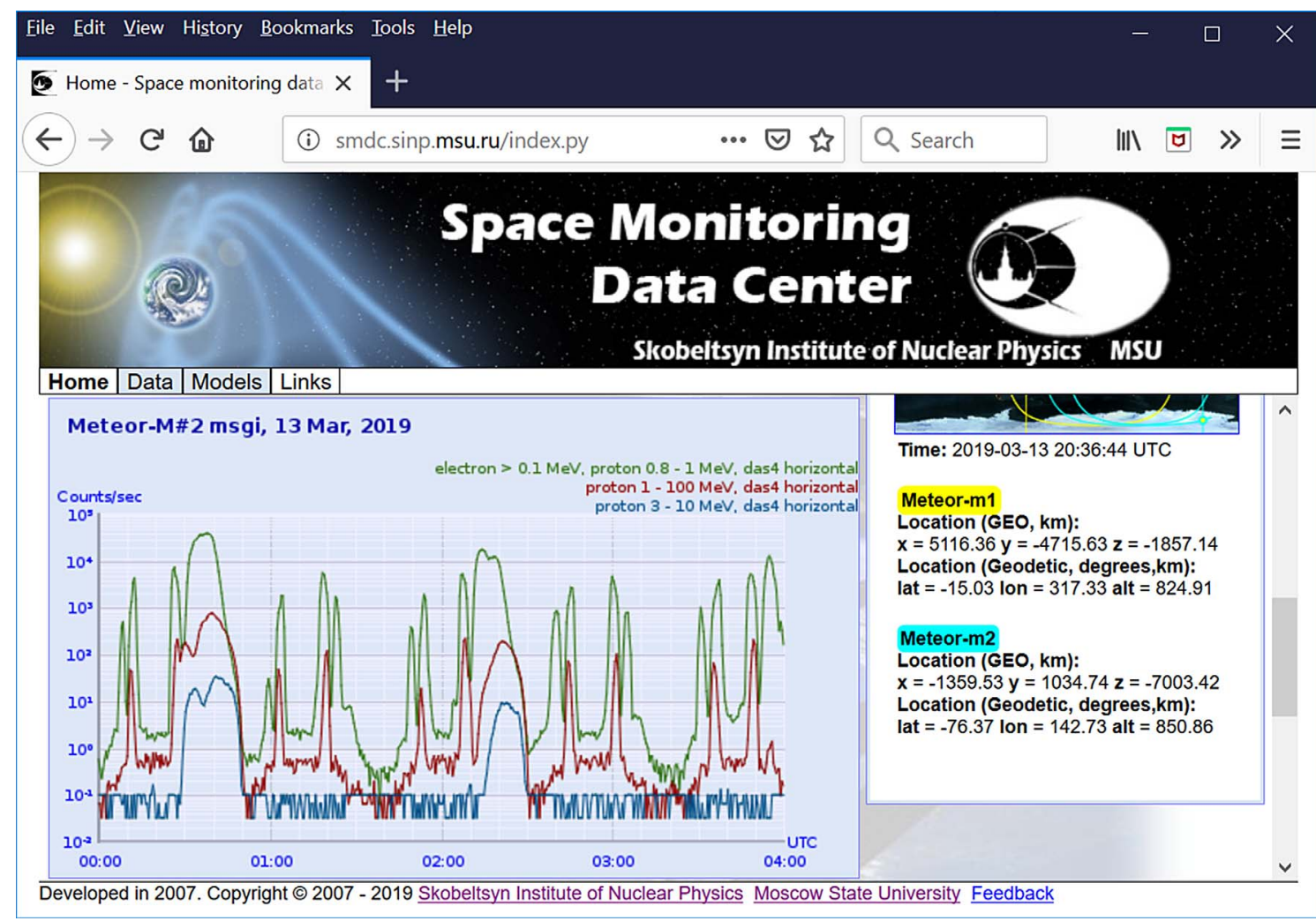

Fig. 1. Main page of SMDC web-site (http://smdc.sinp.msu.ru/index.py).

opportunity to provide the complex analysis of the data "from Sun to Earth" including solar activity (Sect. 4), magnetospheric state (Sect. 5) and radiation conditions (Sects. 6 and 7). SMDC operational models presented here are described in more detail in separate papers (Kalegaev, 2011; Shugay et al., 2011; Myagkova et al., 2017). In parallel to presenting the outputs of the Space Weather services the ongoing physical processes are shortly discussed and interpreted in Section 8. Comparison of the results obtained with third party data allows to validate the operational models implemented in SMDC web-site. Such analysis gives the way to improve the reliability and increase the accuracy of the space weather applications.

\section{Overview of MSU's SMDC data, models and operational applications}

SINP MSU provides access to near real-time space experimental data, as well as to operational forecasting models pertaining to space weather phenomena, mainly related to radiation conditions, through interactive applications of two SMDC web-sites http://smdc.sinp.msu.ru/ and http://swx.sinp. msu.ru. Both web-sites use the same database which works under PostgreSQL. While http://smdc.sinp.msu.ru/ is aimed to give simple access to data collected in SMDC data base, http://swx.sinp.msu.ru web-site is intended to describe current space weather conditions using near real-time information coming from different data sources or from operational models.
Particle fluxes measured on-board several Russian weather satellites and scientific space missions can be obtained through http://smdc.sinp.msu.ru/ web-site. There are, mainly, the historical data from the past space experiments. Figure 1 represents the main page of http://smdc.sinp.msu.ru/ web-site. Table 1 shows data availability for some missions presented at this SMDC web-site.

Current solar activity, geomagnetic and radiation conditions in the Earth's magnetosphere and heliosphere can be accessed in the near real-time mode using SMDC' Space Weather web-site (http://swx.sinp.msu.ru). As Space Weather resource, this internet service uses data coming from different active space missions, like Meteor-M2, Electro-L2, GOES-14, GOES-15, SDO, DSCOVR and so on. From the other hand, SWX data services allow also to get and analyze historical data collected in SMDC database. Advanced graphical applications give possibility to compare data coming from different data sources. In the rest of this paper we will consider only SMDC' space weather web-site (http://swx.sinp.msu.ru), referred below as SWX. Figure 2 shows its main page representing the current conditions in the Earth's environment: Solar X-ray flux, Kp index of geomagnetic activity, $10 \mathrm{MeV}$ proton flux and subrelativistic electron flux at GEO with 5 min update rate. Now, the data sources are GOES-14, GOES-15 and Electro-L2 satellites as well as Potsdam's Geophysical Center.

SWX web-site collects the data coming from different sources: satellites, on-ground stations, data centers, quantitative models. Special software, Satellite Data Downloading System (SDDS), is developed to collect and process the data. SDDS services convert the primary data coming from different 
Table 1. Satellite data availability at SMDC SINP MSU.

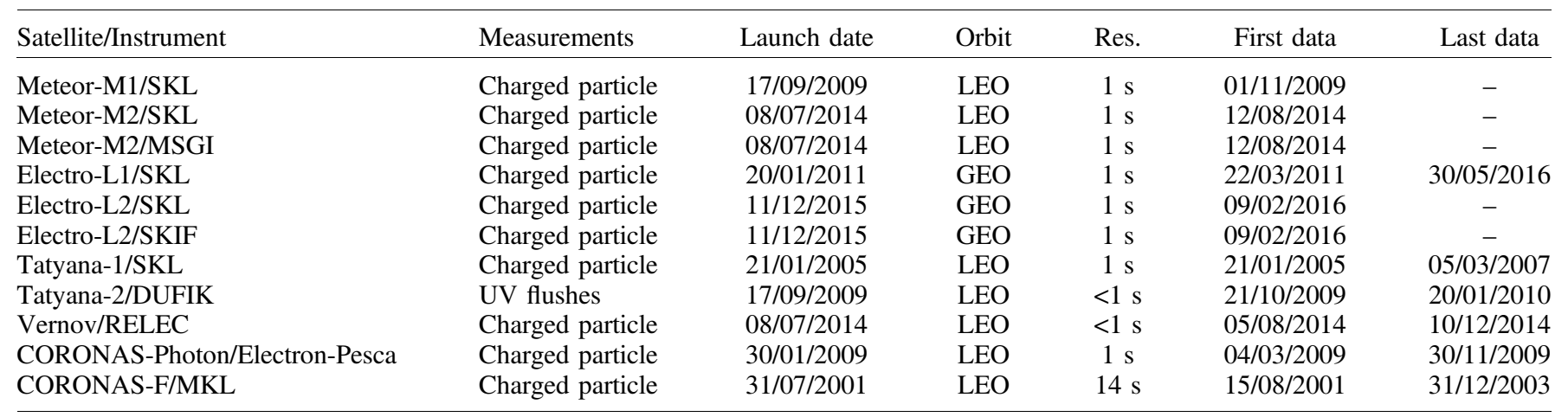

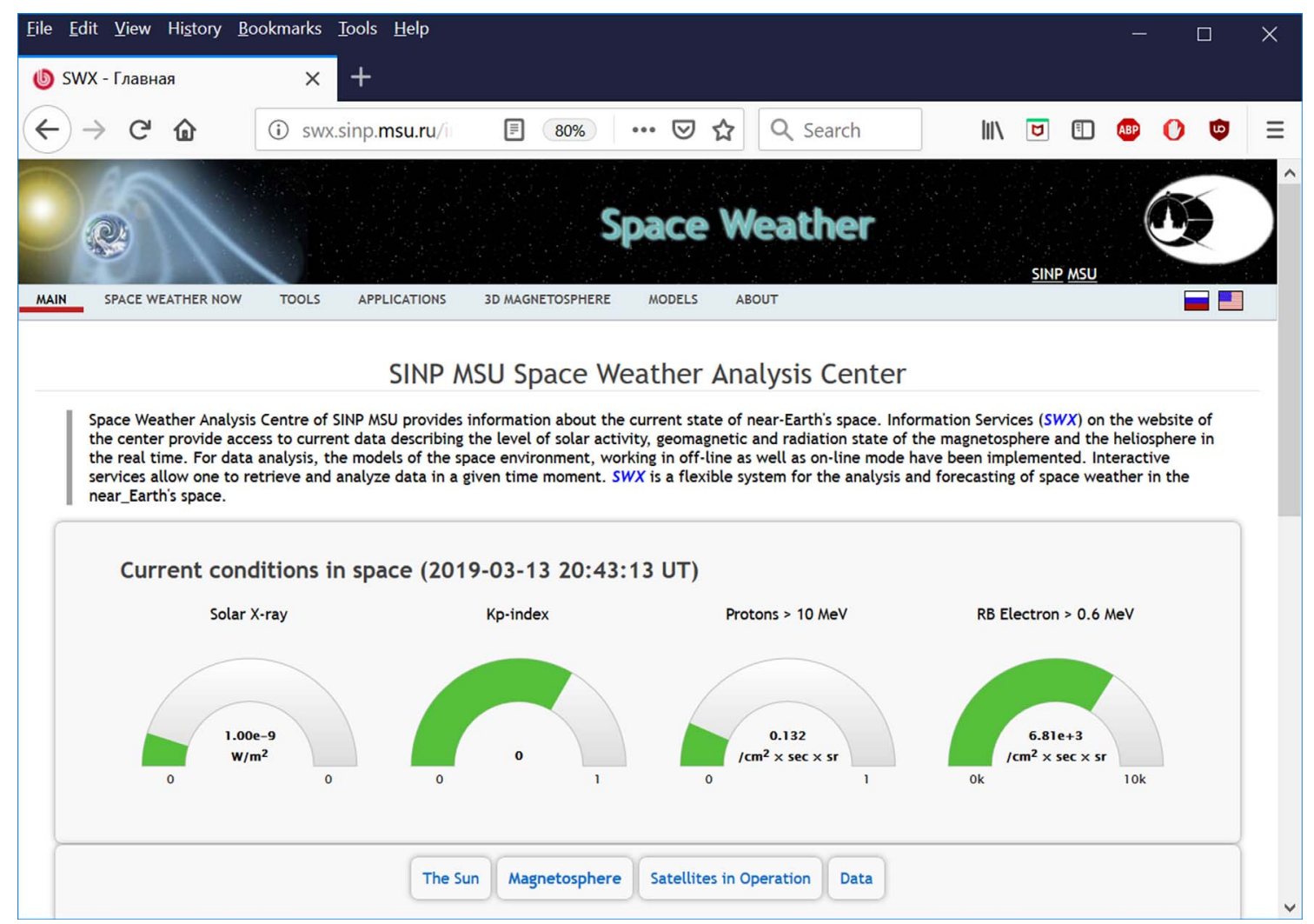

Fig. 2. SMDC' SWX web-site main page (http://swx.sinp.msu.ru/index.php?lang=en).

sources into unified format and load data into different tables of data base. There are two ways for users to retrieve data from SMDC storage: either request data directly from their programs using the RESTful API of SDDS or retrieve data interactively using Web-applications. One of the advantages of SMDC data system is that the system allows users to retrieve data from different satellites as a single array of time series with different cadence. This feature is very useful in data analysis and enables physicists to focus on their work and not to spend time on data preparation. First of all, it reduces the effort in collecting data sets from different sources and converting them into a unified format suitable for further use. The latter work is usually time-consuming and error-prone. Secondly, when various data sets are put together, they give a more precise overall picture of the subject to be studied. Being presented together, these data sets can be used for measurements crosscalibration, to diagnose errors in measurement of the satellite instruments.

Operational applications on Space Weather web-site provide real-time analysis and assimilation of experimental data as well as forecasting and post-casting of radiation conditions in nearEarth space. These applications use data from mentioned above spacecraft collected in a single database. Space Weather website represents the space environment physical conditions in the near real-time (page "Space Weather Now", see http:// swx.sinp.msu.ru/weather.php?lang=en). This page gives access to measured data and also to some calculations in terms of SMDC operational services. 
Page "Tools" (http://swx.sinp.msu.ru/tools/ida.php?lang=en $\& \mathrm{gcm}=1$ ) contains several applications that give access to data from SMDC database. Interactive data Analysis Tool (IDA) allows to display the data from different sources (satellite measurements, geomagnetic indices) for a given time interval. One can create templates to generate the standard set of the figures. Some predefined templates are accessible on the IDA Tool page. Hourly averaged data are used in this service. Data Visualisation and Analysis Tool (DaVisAT) gives access to satellite and on-ground data with different time resolutions. Sun Viewer (SunView tool) is catalogue of hourly Sun images obtained by SDO/AIA for different wavelengths. All these instruments can be used to carry out the analysis of the space weather conditions for a given time interval. IDA and DaVisAT tools were used to create the most of the figures presented in this paper.

Page "Applications" (http://swx.sinp.msu.ru/apps/geospace_ now.php?lang=en\&gcm=1) gives access to data from the models and services that working at SMDC in operational mode. "Geospace Now" gives a brief summary on Space Weather conditions (Sun X-ray, Geomagnetic and Radiation conditions) now and during the current week. "Heliosphere" gives forecast of quasi-stationary solar wind velocity based on image analysis of the Sun from the SDO/AIA space observatory. "Magnetosphere" gives access to several models that forecast the physical conditions in the near-Earth's space. There are short-term $(\sim 1 \mathrm{~h})$ forecasts of Dst, solar wind plasma and IMF at the Earth's orbit, magnetopause subsolar distance, as well as middle-term (1 day) forecast of relativistic electron fluences at the geostationary orbit. Page "3D Magnetosphere" allows to reproduce magnetospheric magnetic field structure by Alexeev et al. (2001), Kalegaev (2011) in the real-time as well as for a given time moment. Page "Models" is intended to give access to the interactive models that can be used for scientific investigations but does not reach the "operational" level.

\section{Overall space environment conditions in near-Earth Space}

Analysis and cross-comparison of data from multi-satellite measurements as well as magnetospheric modeling can provide information concerning the mechanisms responsible for the different dynamical processes taking place in the Earth's magnetosphere. Space environment conditions and its effects during the time period February 14-March 5, 2014 are presented using tools available on the SMDC Space Weather website. Time profiles of basic parameters describing the influence of the Sun on the Earth's magnetosphere (solar emission, solar wind and IMF) as well as parameters representing the magnetospheric response (relativistic electron fluxes, Dst-variation) are shown in Figure 3.

These pictures can be obtained using IDA tool of the SWX website (http://swx.sinp.msu.ru/tools/ida.php?lang=en $\& \mathrm{gcm}=1)$. During the considered time interval, the radiation environment in near-Earth space was very unstable, two complex SEP events (Fig. 3d) and several magnetic storms (Fig. 3f and g) occurred in the Earth's environment. These phenomena are related to solar flares observed by GOES-15 X-Ray instrument (see Fig. 3a).
The sources of the geomagnetic disturbances were the solar wind pressure pulses (Fig. 3b) in combination with the southward IMF excursions (Fig. 3c). Several HSS of solar wind from coronal holes $(\mathrm{CH})$ and coronal mass ejections (CMEs) produced the conditions in solar wind responsible for geomagnetic disturbances. As a consequence of the high geomagnetic activity influenced by solar wind variations, several dropouts of relativistic electron fluxes occurred during the considered time period (Fig. 3e). Solar wind pulses under different IMF directions provided magnetospheric responses of various types.

Dynamics of the magnetospheric parameters during the considered time interval are presented using the operational models in Section 5.

\section{Coronal hole sources of near-Earth solar wind streams}

Prediction of the solar wind stream characteristics is important for early determination of physical conditions in near Earth space. To predict the arrival of HSS from $\mathrm{CH}$ and CMEs to Earth, one needs first to determine the coronal sources of these streams, which can be identified by specific signatures. To select the quasistationary HSSs and interplanetary CME that arrived at near-Earth orbit from February 14 to March 5, 2014 we used images obtained by SDO/AIA (Lemen et al., 2012) (https:// sdo.gsfc.nasa.gov) and SOHO/LASCO (Brueckner et al., 1995) together with information about CME parameters from Coordinated Data Analysis Workshops (CDAW) (https:// cdaw.gsfc.nasa.gov), Solar Eruptive Event Detection System (SEEDS) (http://spaceweather.gmu.edu/seeds/secchi.php). We used data about the Interplanetary Coronal Mass Ejections (ICMEs) from the catalog by I. Ricardson and $\mathrm{H}$. Cane (http://www.srl.caltech.edu/ACE/ASC/DATA/level3/icmetable2. htm) and GMU CME/ICME List by Phillip Hess, Jie Zhang and Yutian Chi (http://solar.gmu.edu/heliophysics/index.php/GMU_ CME/ICME_List).

The sources of recurrent HSSs are $\mathrm{CH}$, which can be identified as large regions of open magnetic field lines or seen in the EUV wavelength range as large dark areas (Fig. 4). Figure 4 is a synoptic map for CR 2147. We constructed this map from the hourly images in the channels $19.3 \mathrm{~nm}$ obtained by SDO/AIA after drawing of the $\mathrm{CH}$ contours using a threshold algorithm (Shugay et al., 2011). During the period under consideration, several $\mathrm{CHs}$ passed through the central meridian. We used the EUV-imaging based empirical model (Shugay et al., 2011, 2014) to predict the speed and time of arrival of HSSs associated with $\mathrm{CHs}$ at near-Earth orbit. Operational service at SMDC web-site (http://swx.sinp.msu.ru/models/ solar_wind.php?gcm=1\&lang=en) allows to see the results of an online HSSs speed forecast at L1 point based on EUVimages. The arrival time of the HSSs from the two separate parts of the mid-latitude positive polarity $\mathrm{CH} 1$ were estimated February 17-21 and 21-26. The SW stream from the $\mathrm{CH} 2$ was expected from February 28 to March 3 . The estimated maximum speed of the HSSs were 462,435 and $454 \mathrm{~km} \mathrm{~s}^{-1}$, respectively for three intervals (Fig. 5). The difference in the observed and predicted speed and arrived time of the HSSs is connected with the forecast errors and the presence of the ICMEs, the arrival of which is not predicted by EUV-imaging 


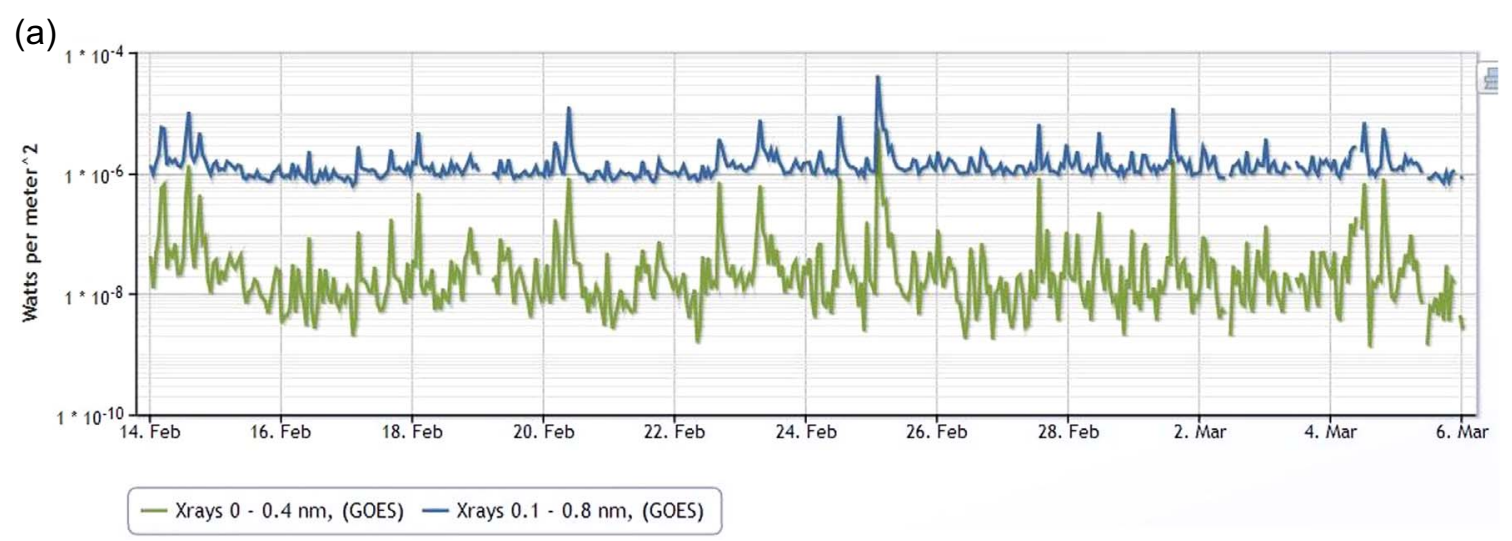

(b)

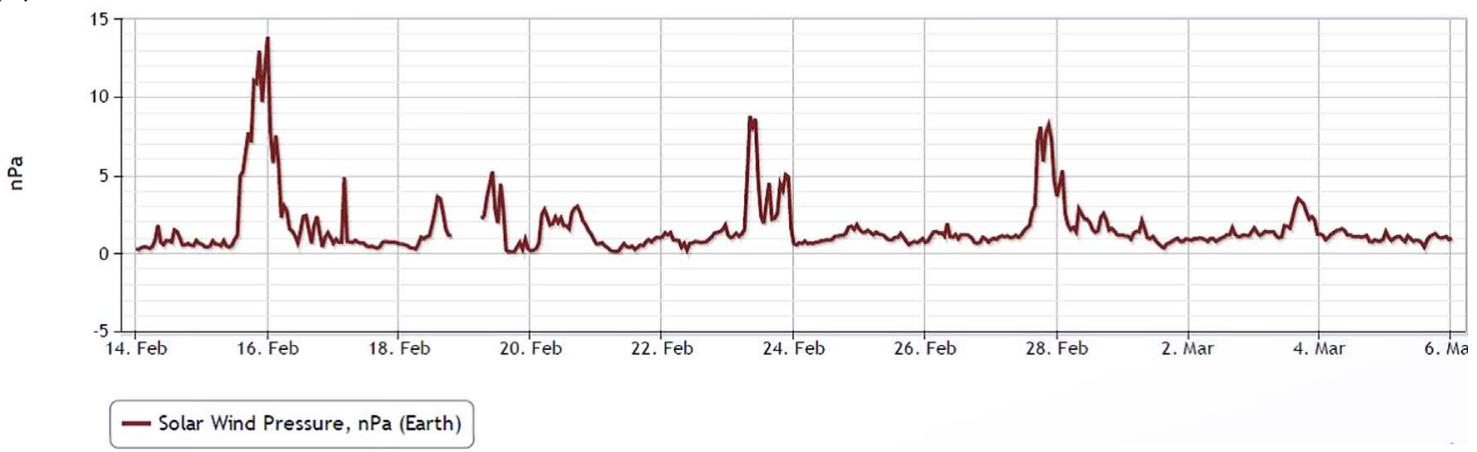

(c)

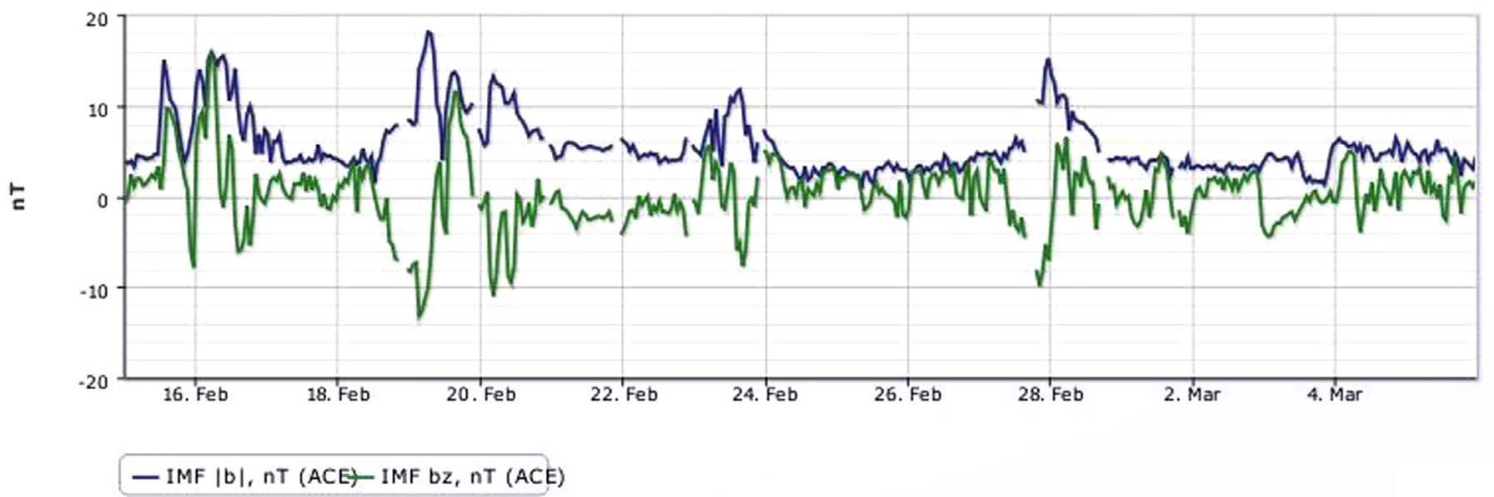

(d)

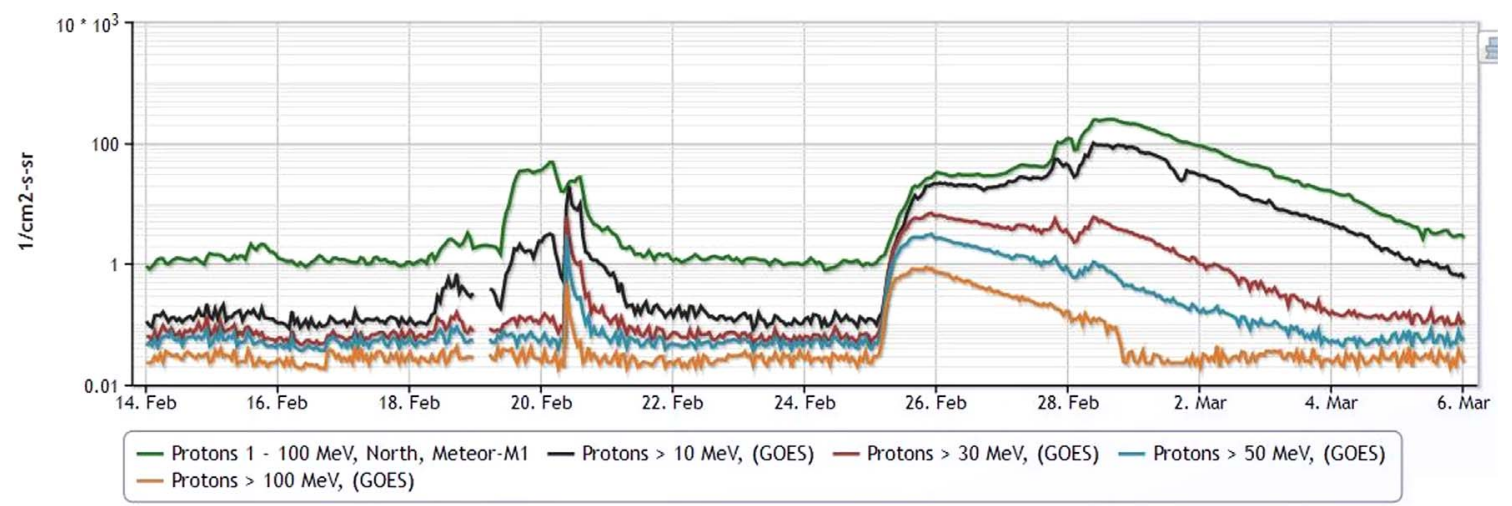

Fig. 3. Time profiles of (a) Sun's X-ray fluxes, (b) solar wind pressure, (c) the magnetic field value and the IMF Bz-component, (d) SEP fluxes, (e) energetic electron fluxes, (f) the Dst-index, and (g) Kp-index (http://swx.sinp.msu.ru/tools/ida.php?gcm=1). 
(e)

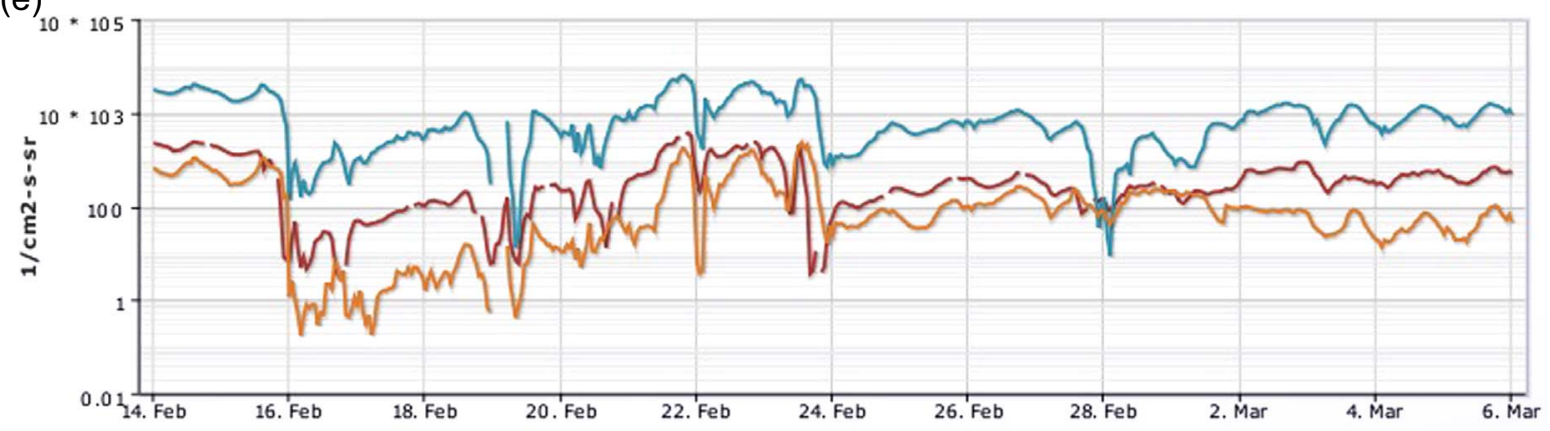

- e > 1.3, p 1.3-95 MeV(Electro-L4)Electrons > 0.8 MeV, (GOES) Electrons > $2 \mathrm{MeV}$, (GOES)

(f)

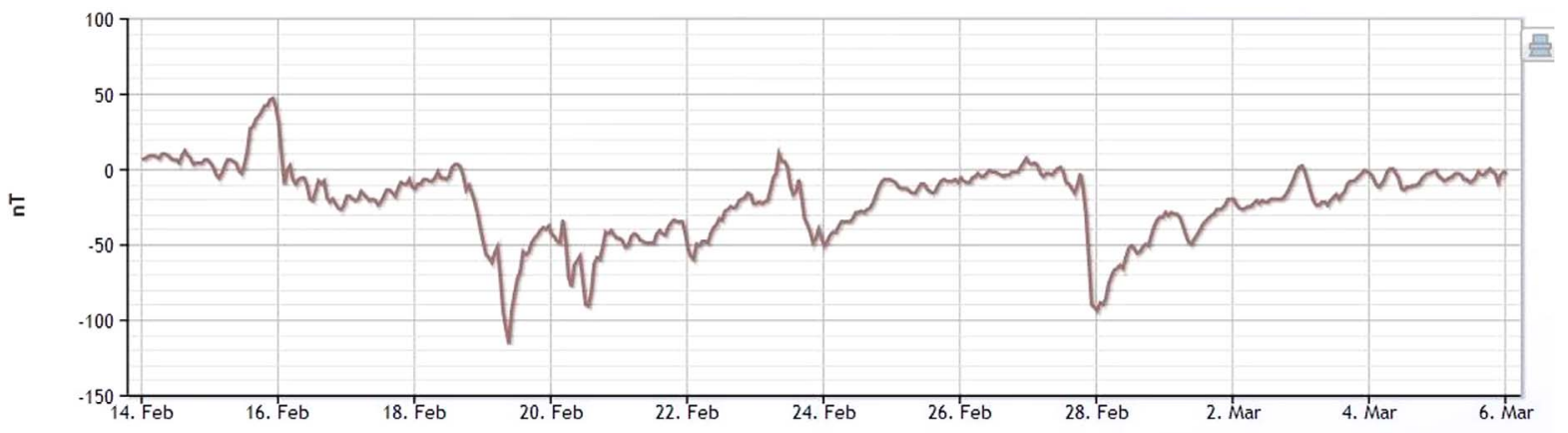

\section{- Dst}

(g)

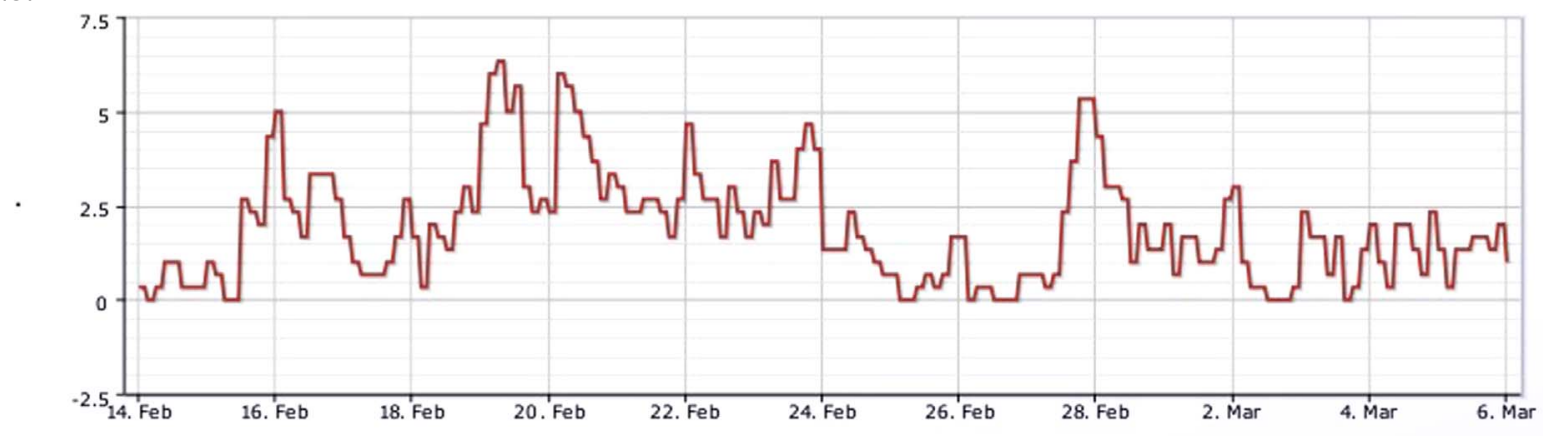

$$
-\mathrm{Kp}
$$

Figure 3. Continued.

based model. ICME can be associated with CME due to flares in the active regions, eruptions by the prominence and are capable of causing strong geomagnetic disturbances. In the separate paper we will consider the CMEs that could have affected the physical conditions in near-Earth space during the period under consideration. It should be noted that during the maximum phase of the solar cycle (2014 year) the forecast of quasi-stationary solar wind streams gives the worst results compared to other years 24 solar cycles. This is reflected in the paper (Reiss et al., 2016), that presents the results of the forecast by Empirical Solar Wind Forecast (ESWF) and Wang-Arge-Sheeley (WSA) models for the period 2011-2014.
Several CHs and CMEs related both to flares and to the eruption of prominences can be associated with the sources of SW streams at $1 \mathrm{AU}$ during the period under consideration. The interaction of different SW streams can lead to the increased geoeffectiveness of SW streams observed in the near Earth space. Analysis of SDO/AIA images allows to determine the sources of quasistationary HSSs and to predict their speed at the Earth's orbit. The current model implemented in SWX does not predict the arrival time and speed of transient streams now. Among the future tasks, there is the inclusion of the model which can predict the speed and arrival time of an ICME in the automatic online SW speed forecast scheme. 


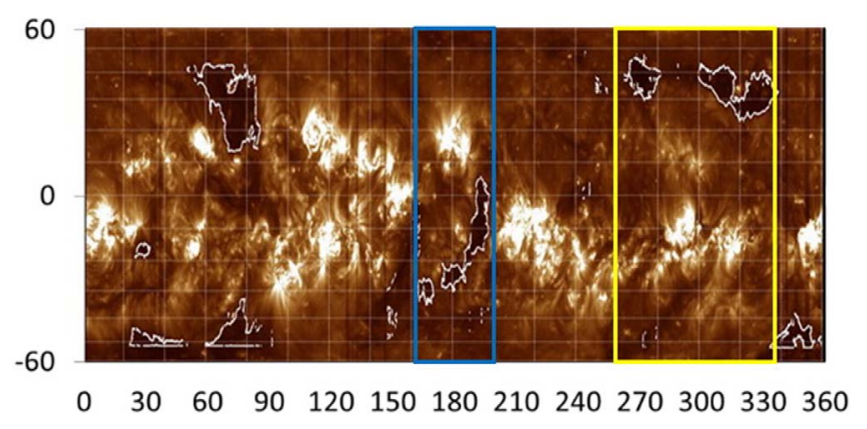

Fig. 4. Synoptic map with $\mathrm{CHs}$ areas constructed from the EUV images in the channels $19.3 \mathrm{~nm}$ obtained by SDO/AIA during the period from 11 February to 9 March, 2014 (CR 2147, time is directed from right to left). The two separate parts of the mid-latitude positive polarity $\mathrm{CH} 1$ were observed during 12-18 February (yellowrectangle) and the equatorial negative polarity $\mathrm{CH} 2-23-26$ February (blue rectangle).

\section{Geomagnetic activity}

Solar activity impacts the magnetosphere in several ways. Geomagnetic response is related to solar wind variations due to HSS or CME propagation. Geoeffective (southward) IMF direction accompanied by solar wind pressure pulse can lead to a magnetic storm. The level of geomagnetic disturbances depends on the relationships between solar wind parameters and IMF in the near-Earth space. SMDC space weather operational service based on processing of solar FUV images allows middle term forecasting the solar wind velocity in the Earth's environment with prediction time about of 3 days ahead (see Sect. 4). On the other hand, spacecraft measurements in the solar wind flow at L1 point give possibility of the short term (about of $1 \mathrm{~h}$ ) forecasting of plasma/magnetic field parameters at the Earth's orbit as well as of geomagnetic and radiation conditions in the Earth's magnetosphere.

\subsection{The magnetopause stand-off distance forecast}

Physical conditions on Earth's orbit can be obtained propagating solar wind parameters from L1 point (where they are measured by ACE or calculated based on Sun observations in terms of the model like that presented in Sect. 4) to the Earth's location. A simple convection delay or "phase front" method described by Pulkkinen \& Rastätter (2009) was used at SMDC as an operational service to describe solar wind and IMF propagation from ACE position to the Earth environment. Figure $6 \mathrm{a}-\mathrm{c}$ compares solar wind parameters (IMF Bz, density and velocity), calculated by SMDC model and those from OMNI database. Correlations for results are 0.93 for density, 0.88 for $\mathrm{Bz}$ and 0.98 for velocity. Solar wind parameters at the Earth's orbit appear in the OMNI database after half a year. Unlike OMNI database, SMDC gives possibility to get solar wind data near the Earth in real time.

SMDC service makes it possible to calculate in real time the magnetopause stand-off distance on the base of some existing magnetopause model (http://swx.sinp.msu.ru/apps/rss/index. php?gcm=1). Taking into account that solar wind has to spend about $1 \mathrm{~h}$ (it depends on solar wind speed value) to reach the Earth's magnetosphere from L1, the calculations from Kuznetsov \& Suvorova (1998) or Shue et al. (1998) models can provide short-term forecast of the magnetopause stand-off distance. The time profile of the magnetopause stand-off distance calculated by Shue et al. (1998) model during February 14-March 5, 2014 is presented on Figure 7.

\subsection{Geomagnetic field structure forecast by the A2000 magnetospheric paraboloid model}

Variations of the magnetospheric structure and the associated charged particle motion in near-Earth space are controlled by solar wind. Understanding the magnetospheric magnetic field dynamics under solar wind influence is one of the key issues of space weather. Dynamical magnetospheric models make it possible to predict the variations of the magnetic field and charged particle populations in the Earth environment based on the solar wind parameters forecast (see Alexeev et al., 1996, 2003; Kalegaev \& Makarenkov 2008; Ganushkina et al., 2010; Kalegaev, 2011; Tsyganenko \& Andreeva 2015 and references therein). Both, empirical-type (Tsyganenko 2002) and so-called first-principle-type models (Alexeev et al., 2003) reproduce magnetic field changes due to solar wind influence. Although the modern MHD models allow to obtain much more

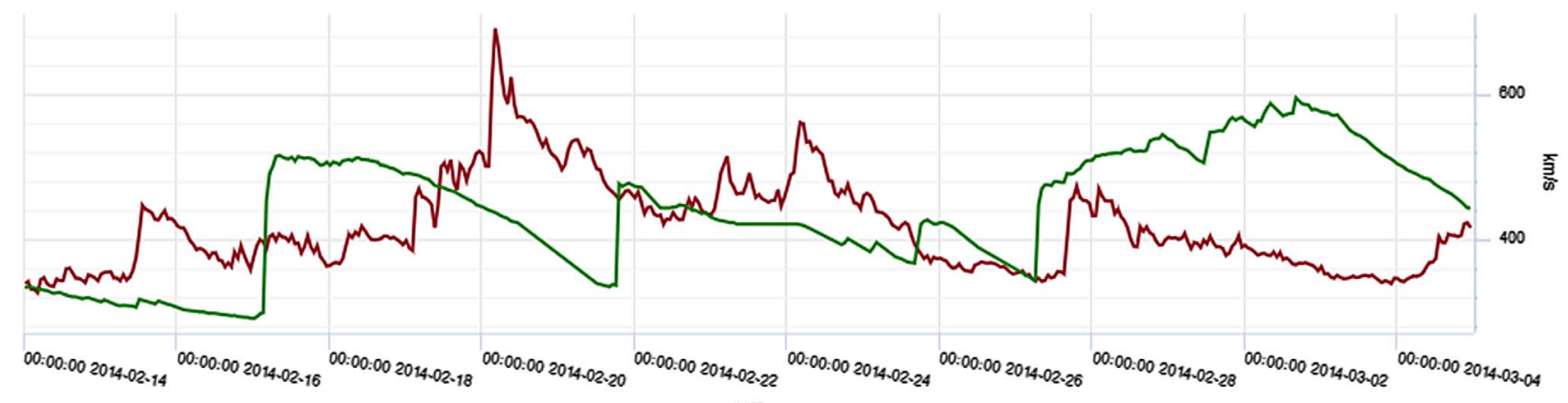

UT

- $v_{s w}\left(A C E\right.$, SWEPAM Corrected) $[\mathrm{km} / \mathrm{s}] \quad-V_{s w}($ by SDO 193A) $[\mathrm{km} / \mathrm{s}]$

Fig. 5. The green curve is the quasistationary HSSs speed predicted by EUV-imaging based model; the red curve is the observed SW speed; the black dot-lines relate to the arrival of a ICME shock at Earth (http://swx.sinp.msu.ru/models/solar_wind.php?gcm=1\&lang=en). 
(a)

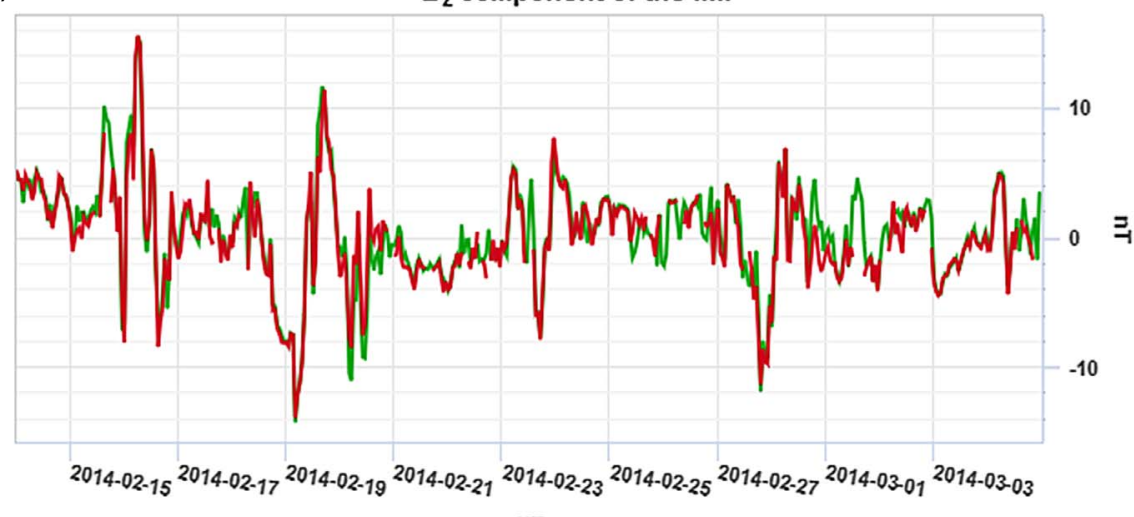

UT

\section{- GSM B $B_{\mathbf{z}}[\mathrm{nT}]-\mathrm{GSM} \mathrm{B}_{\mathbf{z}}$ (OMNI) [nT]}

(b)

Solar Wind Bulk Speed

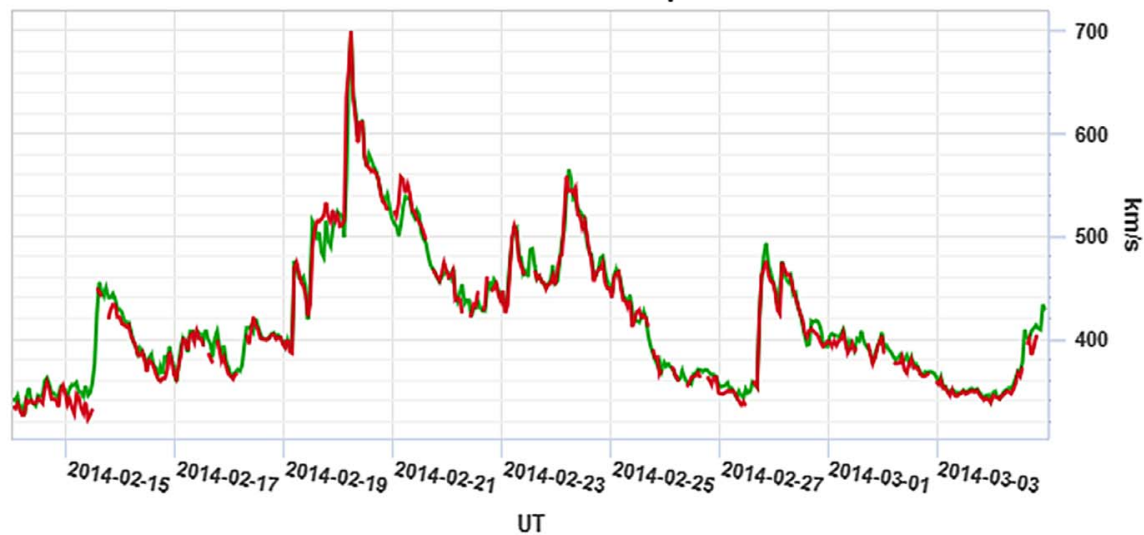

$-\mathrm{v}_{s w}[\mathrm{~km} / \mathrm{s}]-\mathrm{v}_{s w}(\mathrm{OMNI})[\mathrm{km} / \mathrm{c}]$

(c)

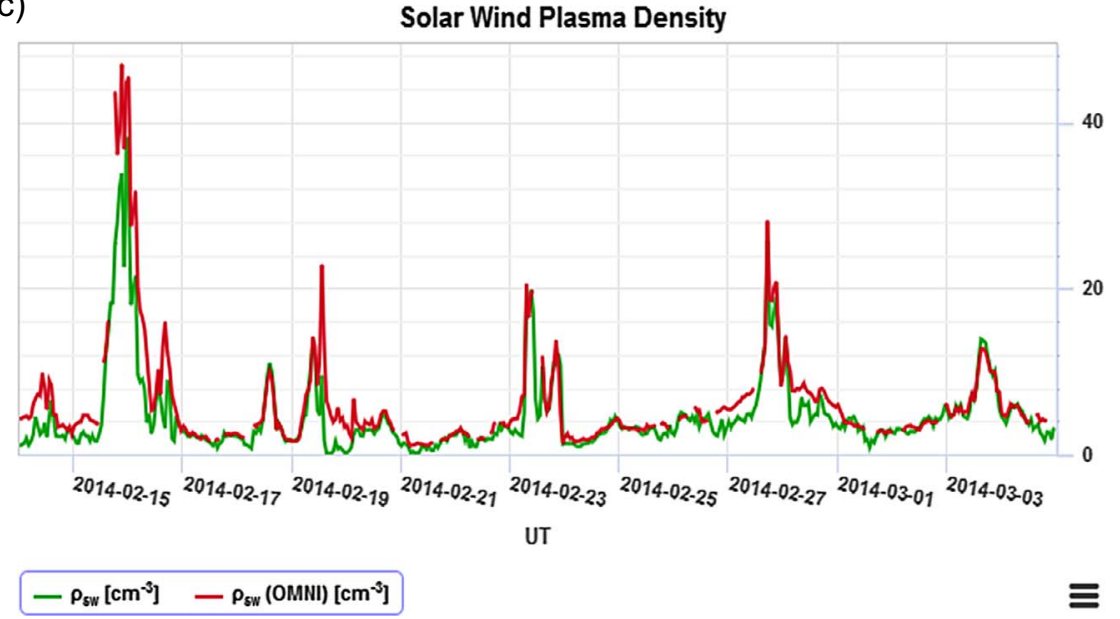

Fig. 6. Time profiles of (a) the IMF Bz-component, (b) solar wind density, and (c) speed calculated by SMDC model (green) and obtained from OMNI database (http://swx.sinp.msu.ru/apps/solar_wind.php?gcm=1\&lang=en, red).

extended information about the physical conditions in Earth environment including magnetosphere - ionosphere - ring current coupling (see e.g., Pembroke et al., 2012), dynamical magnetospheric models are still in great demand. The main advantages of such models are a better time performance in comparison with MHD models and the possibility to describe 


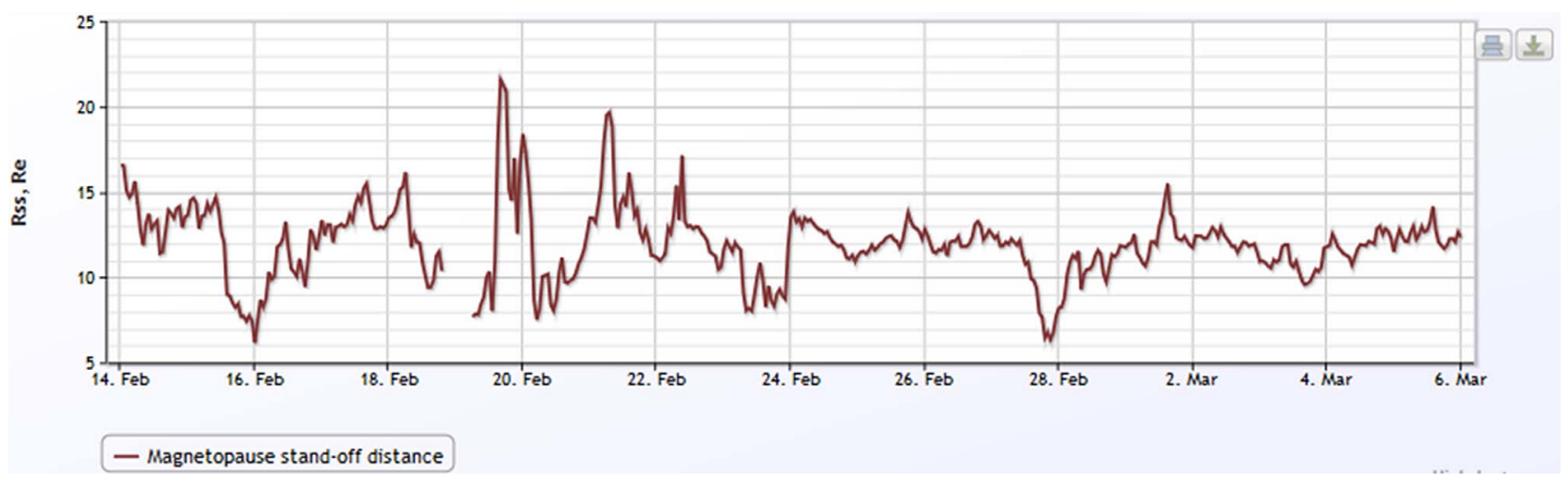

Fig. 7. The time profile of the magnetopause stand-off distance (http://swx.sinp.msu.ru/tools/ida.php?gcm=1).

the dynamics of the main large-scale current systems in the magnetosphere.

The paraboloid model of Earth's magnetosphere A2000 (Alexeev et al., 2001, 2003) is implemented at SMDC to describe magnetic field structure and dynamics in the Earth's environment depending on the empirical data (solar wind parameters and geomagnetic indices, see Alexeev et al., 2003). The storm-time dynamics of the magnetosphere can be reproduced through the temporal variations of the solar wind and IMF upstream of the Earth's bow shock. Figure 8 represents the 3D magnetospheric structure (http://swx.sinp.msu.ru/3d. php?lang=en) calculated in terms of paraboloid model A2000 with the solar wind conditions near the Earth on February 16, 2014 at 00:00 UT, obtained from the solar wind parameters measured by ACE spacecraft at L1 and propagated to the Earth orbit using the approach presented in Section 5.1. Such approach allows to get magnetospheric magnetic field structure with prediction time about of $1 \mathrm{~h}$ depending on solar wind velocity.

\subsection{Geomagnetic activity forecast}

One of the most important space weather parameters is the Dst-index. Its variations describe the level of geomagnetic activity. Prediction of Dst-index is very important task for advance warning of the geomagnetic storm magnitude. For example, Space Research Institute (Moscow) provides real-time predictions of the geomagnetic storm magnitude (Podladchikova \& Petrukovich 2012) that are updated every hour and are published on http://spaceweather.ru.

University of California, Berkeley (http://sprg.ssl.berkeley. edu/dst_index/welcome.html) produces a prediction of Dst index $1 \mathrm{~h}$ ahead using data from ACE spacecraft, based on the modification of the empirical formula of Burton (Burton et al., 1975). The Swedish Space Weather Center (http://src.irf.se/ en/forecasts/) predicts the next hourly value of the Dst index using the recurrent Elman neural network. Patra et al. (2011) provide Dst predictions using physical WINDMI model based on the calculation of ring currents in the magnetosphere-ionosphere system, using input data from ACE spacecraft. Revallo et al. (2014) proposed Dst index $1 \mathrm{~h}$ ahead forecasting model based on artificial neural networks (ANNs) combined with an analytical model of the SW - Earth's magnetosphere interaction.

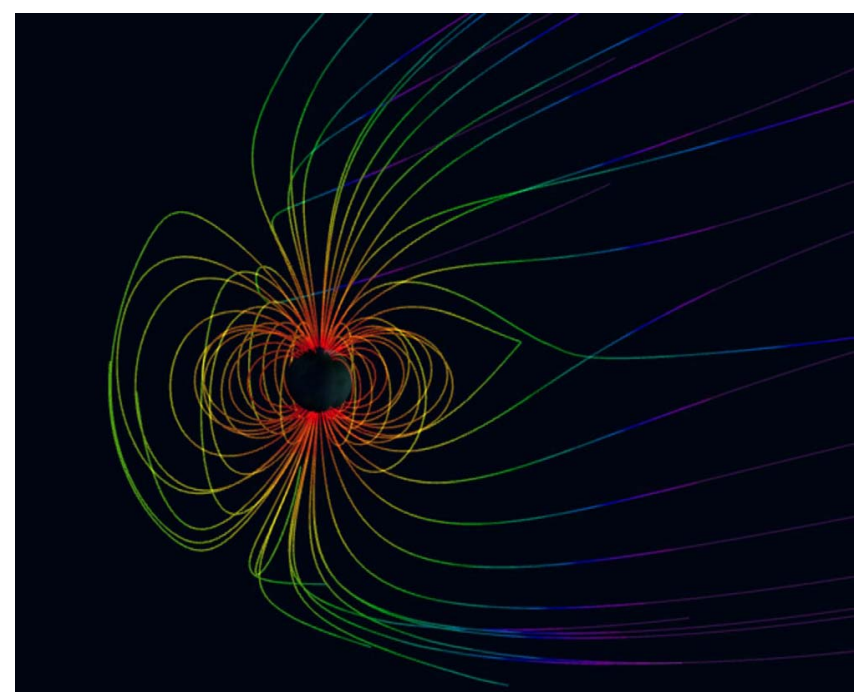

Fig. 8. Magnetic field structure at 00:00 UT on 16.02.2014 (http:// swx.sinp.msu.ru/3d.php?lang=en).

SMDC implements a Dst index prediction service that uses scientific models based on ANN technology (Dolenko et al., 2005; Myagkova et al., 2017). SMDC operational service gives online $1 \mathrm{~h}$ prediction of Dst index using in-situ ACE spacecraft measurements of solar wind and IMF parameters in terms of ANN approach (http://swx.sinp.msu.ru/models/dst.php?gcm=1 \&lang=en). Results of the Dst predictions calculated during period on 14 February-5 March, 2014 are shown in Figure 9. Figure shows that both geomagnetic disturbances as well as sudden commencement on 15 February 2014 have been reproduced with sufficient accuracy.

\section{Solar proton events}

The time profiles of energetic proton fluxes during February 14-March 5, 2014 are shown in Figure 3d. Two events were observed during the period studied: on February 20 and February 25, 2014 (ftp://ftp.swpc.noaa.gov/pub/indices/SPE. txt). The first SEP was associated with a solar flare at 


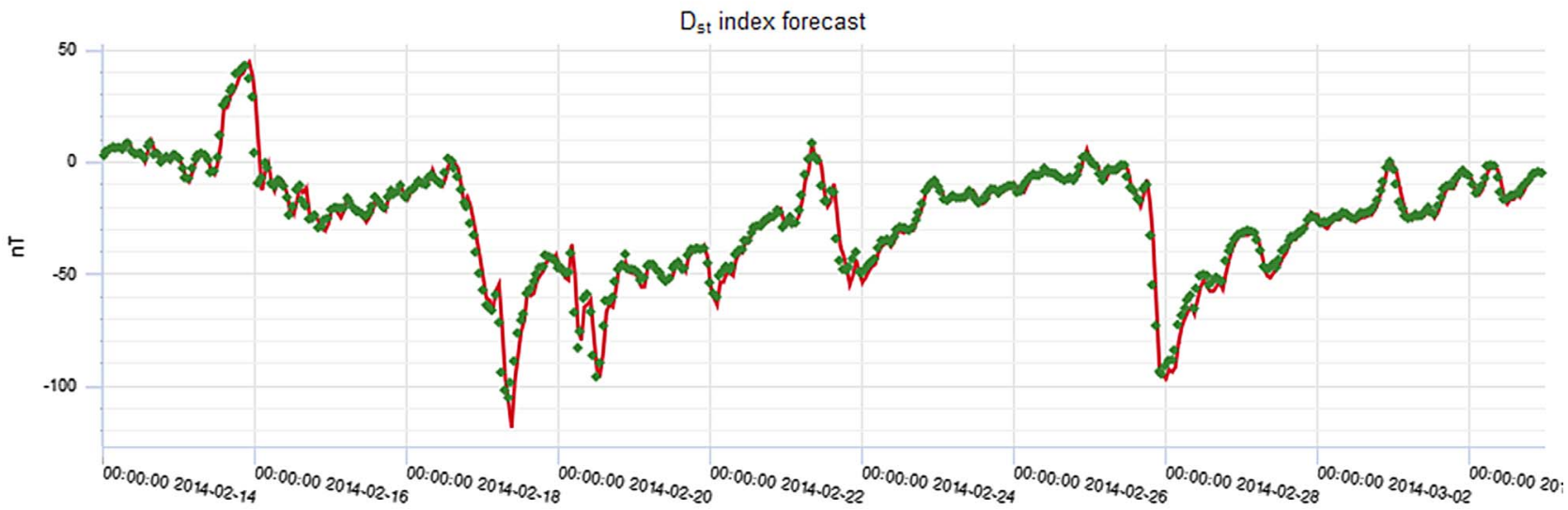

UT

— $D_{s t}[n]$ - D:t forecast, Dst forecast model $[n]$

Fig. 9. The Dst-variation time profiles - measured (red) and forecasted (green) by the artificial neural network (http://swx.sinp.msu.ru/models/ dst.php?gcm=1\&lang=en).

07:55 UT on February 20 (M3; S15W67; AO11976; 22 pfu). The second SPE was associated with a solar flare starting at 00:39 UT and peaking at 00:49 UT on February 25 (X4.9; S12E82; AO11990; 103 pfu). The second SEP event was described in detail in the article (Lario et al., 2016).

Russian spacecraft Meteor-M and Electro-L series have SINP MSU scientific instrumentation on-board to measure SEP fluxes. These data can complement measurements of ACE and GOES spacecraft that are the main source of data about radiation conditions in near-Earth space for space weather centers. Time profiles of data measured by the low-orbiting polar satellite Meteor-M1 and by the geostationary satellite Electro-L1 are presented in Figures 10 and 11, respectively. Meteor-M1 data show the average value over a satellite span on the Northern and Southern Earth's polar cups. Polar satellite measurements give opportunity to study low energetic solar particle fluxes which cannot be measured on geostationary orbits. Separate observations in the Northern and Southern Earth's polar cups are also important during the beginning of an SEP event when particle fluxes are often sufficiently anisotropic and collimated. Asymmetric SEP entries into the northern and southern polar cups can be detected during such events by simultaneous observations of two LEO satellites (Fig. 10).

A set of Solar Energetic Particle Events (SEPE) catalogs of 20-23th solar cycles has been created at SINP MSU (Logachev et al., 2014, 2016) and can be also accessed through the SMDC web-site http://swx.sinp.msu.ru/apps/sep_events_cat/index.php? $\mathrm{gcm}=1 \&$ lang=en).

\section{Relativistic electrons of the Earth's outer RB}

Outer RB relativistic electron flux dynamics is probably the most investigated but incomprehensible problem of magnetospheric physics. The studied period was interesting due to various phenomena occurring in the solar wind that influenced the Earth's magnetosphere (see Sect. 3). These factors affected also outer RB relativistic electron flux dynamics. Electron intensity-time profiles measured at GEO by GOES-13 and Electro-L1 satellites for the studied period are presented in Figure 3e. One can distinguish several distinctly visible intervals in Figure 3e representing different electron fluxes response to interplanetary medium impacts. The basic features are observed by satellites (dropouts on 16-17, 19-21, 23-24 and 27-28 February, 2014 and consequent particle fluxes enhancements) and can be analyzed by SMDC tools together with solar wind plasma parameters and IMF direction and amplitude.

The period of high level solar and geomagnetic activity from February 14 to March 5, 2014 represents multiple examples of RB dynamics that can be better understood using forecasting models which take into account solar wind input data.

\subsection{Forecast of Relativistic Electron Flux at GEO by ANNs}

It should be noted that the processes in the outer electron RB most likely have a nonlinear character. This leads to the fact that outer RB has a certain "memory", i.e., that its instant state is not completely determined by instant values of external parameters, and the duration of "memory" and relaxation processes for RBs are different for various conditions of the Earth's magnetosphere.

Relativistic electrons of the outer RB are sometimes called "killer electrons", as they are very dangerous for electronic devices, in particular, for the microcircuits used in spacecrafts, and can cause a breakdown in their normal operation (e.g., Iucci et al., 2005). Due to continuous growth of the spacecraft total number and also complication and miniaturization of satellite electronics, the number of the failures connected with influence of space weather factors will increase. Therefore, the possible prediction of the reaction of the outer $\mathrm{RB}$ to changes of the physical parameters of IMF and solar wind becomes more and more critical. 


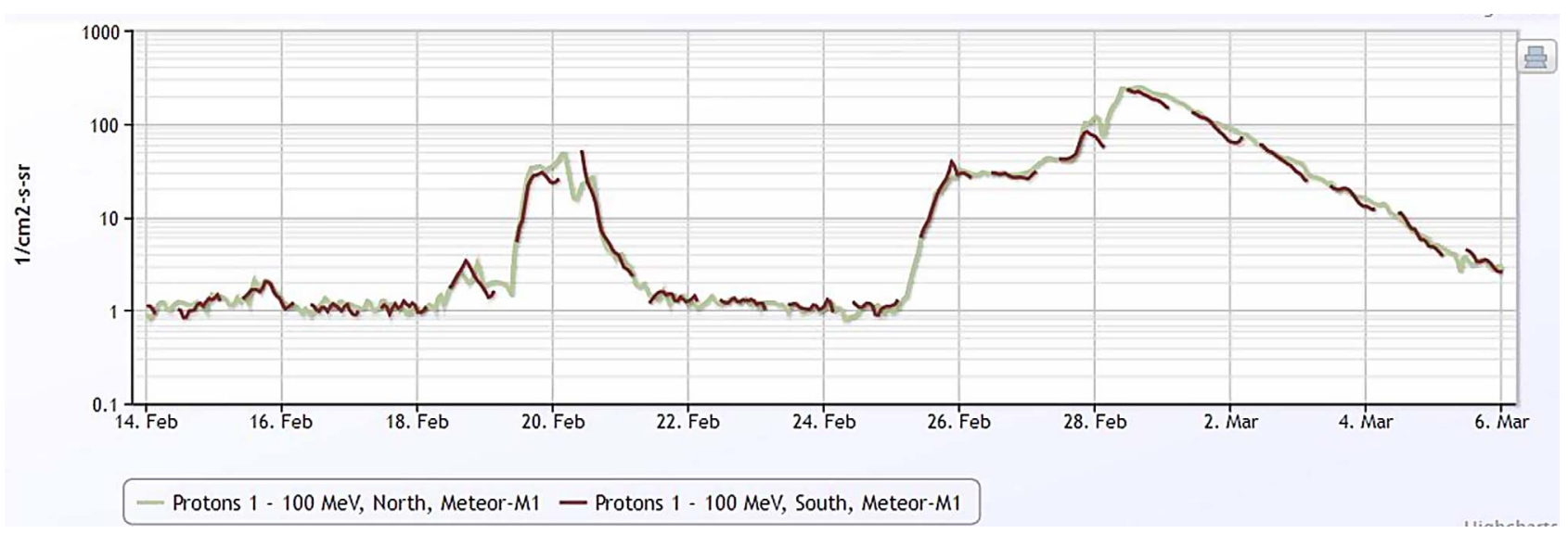

Fig. 10. Time profiles of SEP fluxes measured by Meteor-M1 from February 14 to March 6, 2014.

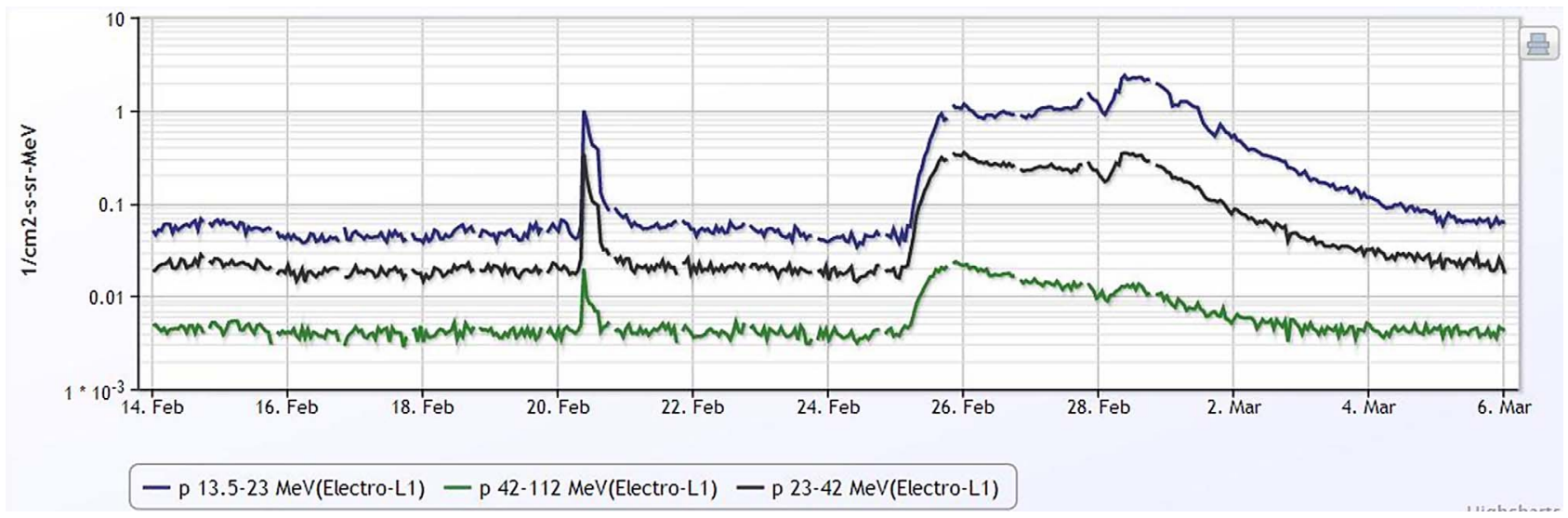

Fig. 11. Time profiles of SEP fluxes measured by Electro-L1 from February 14 to March 6, 2014.

High correlation between electron fluxes at geosynchronous orbit and SW parameters was described back in 1979 (Paulikas \& Blake, 1979), and this is the most important experimental result for prediction. (Baker et al., 1990), demonstrated that integral day values (fluence) of the flux of electrons with energy $>2 \mathrm{MeV}$, measured at geosynchronous orbit, can be predicted 1 day ahead, using a linear filter with solar wind speed as the input. Later, during elaboration of Relativistic Electron Forecast Model (REFM), this method was developed in order to increase the prediction quality and horizon. Prediction carried out with the help of REFM is presented at the portal of Space Weather Prediction Center, http://www.swpc.noaa.gov.

An alternative approach to prediction of relativistic electron fluxes in the outer ERB is based on the use of ANNs. This approach is realized in Koons \& Gorney (1990), Ling et al. (2010) to predict electron flux at geostationary orbit.

Neural network prediction of daily average values of relativistic electron fluences in the outer RB can be found at SMDC' SWX web-site with prediction horizon from 1 to 3 days (http:// swx.sinp.msu.ru/models/rb_electrons/index.php?gcm=1\&lang= en). The operational model uses solar wind and IMF parameters, geomagnetic indices and the measured electron flux as input (Myagkova et al., 2017) to predict electron fluence.
The results of electron flux forecast as well as velocity of solar wind are presented in Figure 12. One can see that the forecast of the electron flux with the aid of the ANN made it possible to predict a large-scale fall of the electron fluxes with energy $>2 \mathrm{MeV}$ to the GEO during the magnetic disturbance on February 16 and their subsequent enhancement. The continuation of the growth of electron fluxes with energy $>2 \mathrm{MeV}$ at GEO during magnetic disturbances on February 18-21 without a characteristic drop in the electron flux in the main phase of the magnetic storm was also predicted correctly. However, response of the relativistic electron flux to the high-speed stream of solar wind and to the geomagnetic disturbance on February 28 was not described correctly: the network predicted a decrease in the electron fluxes standard for the magnetic storm (see Fig. 12). Also, the network erroneously predicted a decrease in electron fluxes on March 4, corresponding to the arrival of the solar wind pressure pulse. Periods of the inaccurate description of the electron fluences are marked by blue boxes in the Figure 12.

This allows us to assume that among the inputs of the artificial neural network, some additional parameters responsible for the dynamics of the fluxes of relativistic electrons in the geostationary orbit are absent now. Cross-comparison of the model 
(a)

4 - 2014-02-14 00:00:00-2014-03-0500:00:00 D

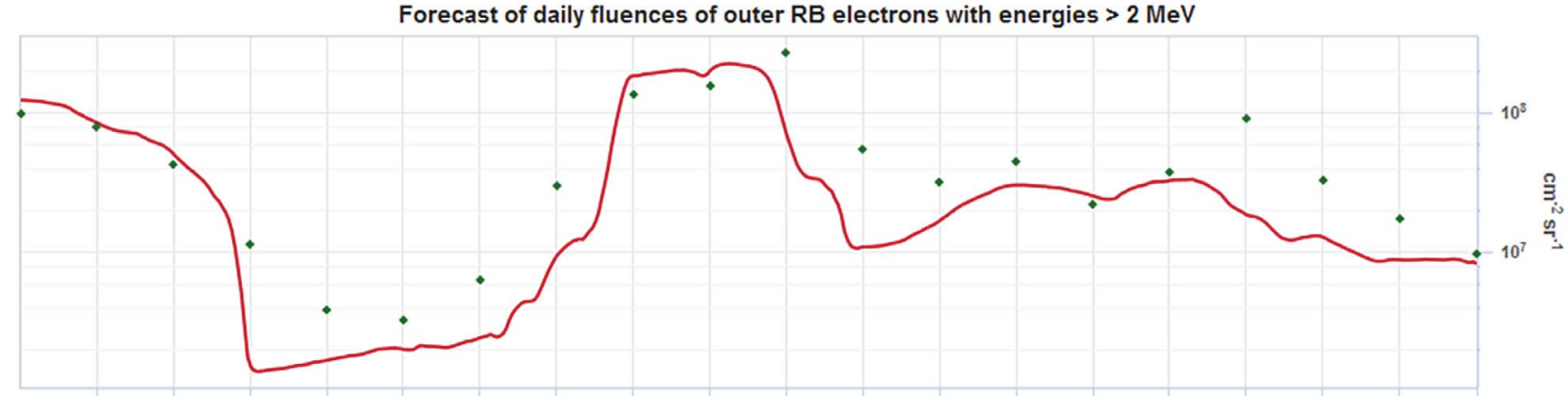

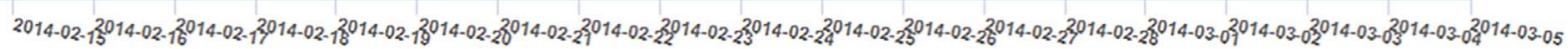

UT

$-E_{\theta}>2.0 \mathrm{MeV} 24$ hours fluence (GOES-P, Particle) $\left[\mathrm{cm}^{-2} \mathrm{sr}^{-1}\right] \quad \forall E_{\theta}>2.0 \mathrm{MeV} 24$ hours fluence, Fluence forecast model [cm-2 $\left.\mathrm{sr}^{-1}\right]$

(b)

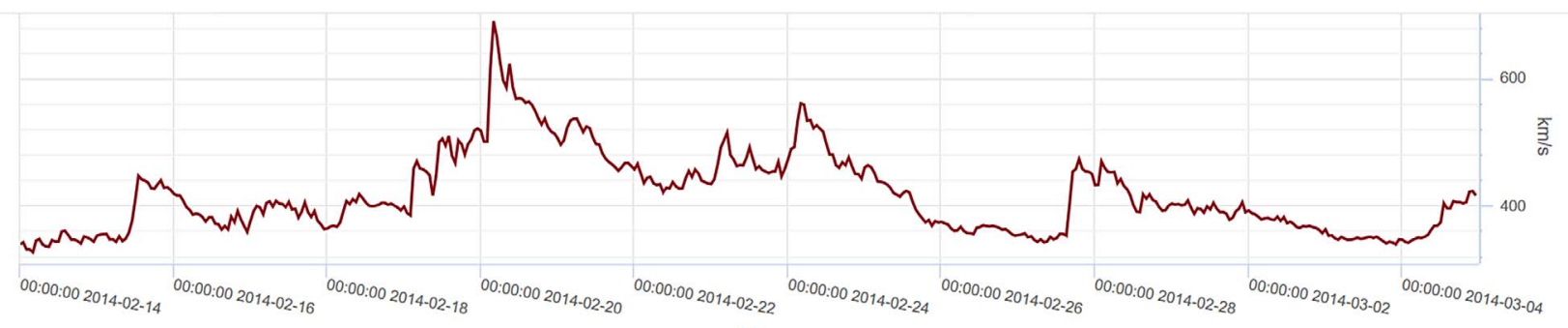

$$
-\mathrm{v}_{\mathrm{sW}} \text { (ACE, SWEPAM Corrected) [km/s] }
$$

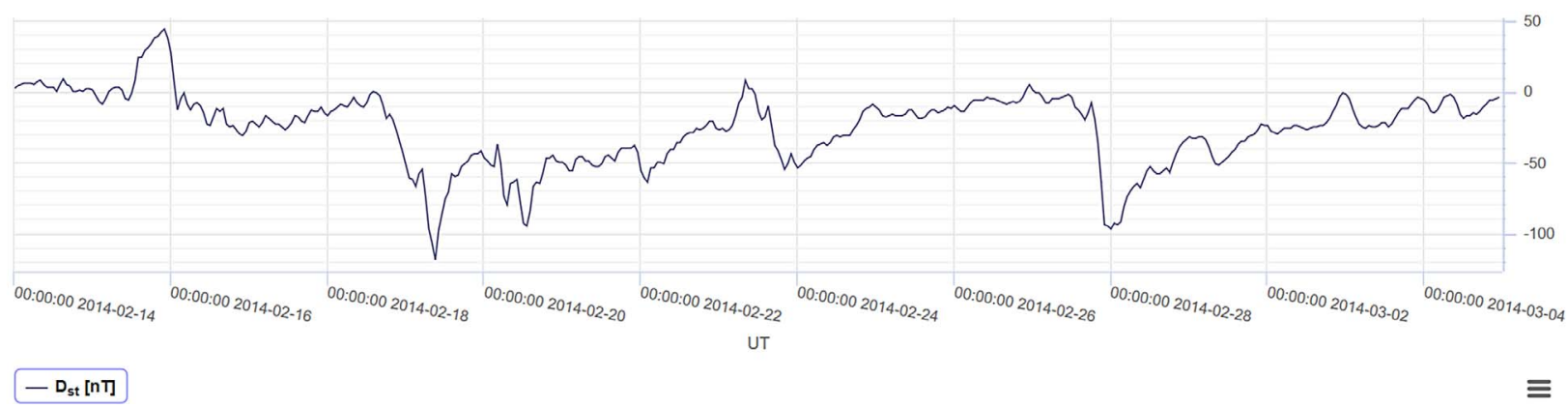

Fig. 12. Forecasting of relativistic electron fluxes - (red - measured, green - forecast) - top panel; velocity of solar wind and Dst - bottom panels. Blue bars shows the periods when dynamics of the relativistic electron fluences was predicted incorrectly.

calculations with the measurements allows us to validate and improve the operational models.

\section{Discussion}

The main goal of this study is to reveal strong and weak aspects of SMDC Space Weather services and demonstrate their possibilities to describe the different manifestations of space weather. Time interval of February 14-March 5, 2014, as noted above, was rich in various phenomena on the Sun, in the solar wind and in IMF and consequently in the Earth's magnetosphere. SMDC tools give possibility to analyse measurement data from different sources as well as modelling data simultaneously in a single workspace. Great abundance of space weather phenomena registered during this time interval gives a good opportunity to reveal advantages and disadvantages of SMDC Space Weather applications. Let us list some phenomena and physical processes that took place in near-Earth space during the studied period of high solar activity:

- The SW pressure pulse under northward IMF on 15 February 2014 caused the magnetopause inward motion and magnetospheric compression up to $\sim 6 \mathrm{R}_{\mathrm{E}}$. One can expect that the outer RB shifted to lower L-shells. Dst increase and dropout of energetic electron flux at GEO with slow recovery have been observed. 
- ICME shock and IMF southward turning on 18-19 February 2014 caused a geomagnetic storm (Dst ${ }_{\max } \sim-120 \mathrm{nT}$ ) accompanied by a decrease and subsequent recovery of the outer radation belt electron fluxes at GEO to prestorm level.

- Specific form of the SEP event on 20 February was formed due to the interaction with interplanetary medium structures (see Sect. 4) that the SEPs met on their way from Sun to Earth. ICME shock with very high bulk speed $\left(>650 \mathrm{~km} \mathrm{~s}^{-1}\right)$ and IMF Bz $<0$ produced a moderate geomagnetic storm (Dst $t_{\max } \sim-80 \mathrm{nT}$ ), Dst-like variation of electron flux were observed at GEO. Electron flux recovery was detected at GEO during the next 2 days.

- Small pressure pulse under southward IMF on 23 February caused a moderate geomagnetic storm $\left(\mathrm{Dst}_{\max }\right.$ $-50 \mathrm{nT}$ ) and electron flux decrease mainly at outer L-shells.

- Classical time profile of solar energetic proton event is observed at GEO on 25 February.

- Small pressure pulse under southward IMF caused a moderate geomagnetic storm $\left(\mathrm{Dst}_{\max } \sim-90 \mathrm{nT}\right)$ on 27-28 February 2014. The magnetopause moved inward up to $\sim 6 \mathrm{R}_{\mathrm{E}}$. Electron fluxes at GEO showed Dst-like variations.

Huge amount of measurement data available during prolonged period of solar and geomagnetic activity gives opportunity for a complete analysis of the various magnetospheric phenomena occurring in Earth environment. SMDC software and databases allow to collect and process data coming from different sources: satellites, ground stations, operational models, neural networks. SMDC services provide crosscomparison of the data obtained from different satellites and from different operational models as well. In general, SMDC operational models presented in this paper describe well measured parameters of the near-Earth's space environment. Simultaneous analysis of experimental data and calculations allow to better understand the mechanisms of Sun-Earth coupling. On the other hand, the measurements-modelling cross-comparison shows some drawbacks or disadvantages of the models that should be eliminated in the future versions of the models.

The analysis of SDO/AIA images allows to determine the sources of solar wind variations at Earth orbit. It was shown that the forecast of the recurrent solar wind streams based only on the coronal hole parameters gives underestimated values of solar wind maximal velocities at $\mathrm{L} 1$ point. The interaction of several SW streams can lead to an increase in their geoeffectiveness. Among the future tasks on SMDC services development is the inclusion into the automatic online solar wind speed forecast scheme of the model that can predict speed and arrival time taking into account parameters of the CME.

The comparison of the ANN RB forecasting model output with the empirical data at GEO showed that the response of the relativistic electron flux on the high-speed stream of solar wind is not always predicted correctly. We assume that among the inputs of the artificial neural network, some additional parameters responsible for the dynamics of the fluxes of relativistic electrons in the geostationary orbit should be taken into account.

One of the main goals of this paper has been to reveal properties of SMDC services that could be improved in the future.
Comparison of the model predictions with the measured data during February 14-March 5, 2014 period gives possibility to validate the models, outline their advantages and limitations and propose the ways to improve them. Their further validation and improvement should be continued based on future analysis, comparison with the other models and additional case studies.

\section{Conclusion}

SMDC of Moscow State University provides mission support for Russian satellites and gives operational analysis and forecasting of radiation conditions in space. SMDC Space Weather web-site (http://swx.sinp.msu.ru/) gives access to current data about the level of solar activity, geomagnetic and radiation state of Earth's magnetosphere and heliosphere in the near real-time mode. The scientific models of space environment factors have been converted into operational engineering services. They are implemented as space weather Webapplications that provide forecasts of geomagnetic and radiation condition in near-Earth space.

The SMDC web-site provides:

- three to five days ahead forecast of the parameters of high-speed solar wind streams based on the coronal hole' parameters;

- short-term forecast of solar wind parameters and IMF at the Earth's orbit by ACE (or DSCOVR) measurement;

- online prediction of Dst index 0.5-1.5 h ahead using ANN by the parameters of SW and IMF measured by the ACE spacecraft;

- middle-term forecast of relativistic electron fluxes of the outer RB;

- instant structure of geomagnetic field lines for a given time on the base of the magnetospheric paraboloid model A2000 using measurements of solar wind and IMF as input parameters.

SMDC Space Weather services were used to describe and analyze space weather phenomena that took place during the period from February 14 to March 5, 2014. Prolonged period of high level solar and geomagnetic activity gives opportunity to demonstrate how well SMDC services describe the different aspects of space weather like solar proton events, geomagnetic storms and outer RB variations. Cross-comparisons of the data obtained from different data sources, both satellites and models, make it possible to validate the existing operational services and understand better the ways for their future improvements. In general, SMDC applications describe well the radiation and geomagnetic conditions in space during studied period. On the other hand, one can see also some drawbacks or disadvantages of the models. For example, solar wind velocity midterm predictions based on Sun observations in FUV wavelength range are needed in corrections by adding the prediction of the CME arrival time and velocity at L1, that was not taken into account in the model implemented on SMDC web-site. The model of the relativistic electron fluences sometimes demonstrates unexpected behavior. The possible reason of this is the absence of the important input parameters for the ANN. 
Comparison of the model calculations with the measurements during multiple consequent case-studies gives possiblity not only to better understand the origin of these events and their sources in space but also to determine the forecasting models drawbacks and improve them in future. SMDC team is planning the continuous development and modification of the operational models and space weather services as a natural and necessary process due to unavoidable unexpected situations and other deviations from a standard used in the design of a model.

Acknowledgements. This study was supported by Russian Scientific Foundation Grant No 16-17-00098. We have used the CME catalog that is generated and maintained at the CDAW Data Center by NASA and The Catholic University of America in cooperation with the Naval Research Laboratory. SOHO is a project of international cooperation between ESA and NASA. Also this paper uses data from the SEEDS CME catalog. The SEEDS project has been supported by NASA Living with a Star Program and NASA Applied Information Systems Research. We are grateful for the opportunity to use the results of the simulation obtained by the DBM models. The authors thank data holders for SDO/AIA, SOHO/LASCO, ACE, Van Allen Probes, NOAA/POES and GOES as well as geomagnetic indices data. The authors thank data services CDAW, CACTUS and OMNIWeb. The editor thanks Norma Crosby and an anonymous referee for their assistance in evaluating this paper.

\section{References}

Albertson VD, Thorson JM, Clayton RE, Tripathy RE. 1973. Solar induced currents in power systems: Cause and effects. IEEE Trans Power Appar Syst PAS-92: 471-477.

Alexeev II, Belenkaya ES, Kalegaev VV, Feldstein YI, Grafe A. 1996. Magnetic storms and magnetotail currents. J Geophys Res 101: $7737-7748$.

Alexeev II, Kalegaev VV, Belenkaya ES, Bobrovnikov SY, Feldstein YI, Gromova LI. 2001. Dynamic model of the magnetosphere: Case study for January 9-12, 1997. J Geophys Res 106(A11): 25683-25693.

Alexeev II, Belenkaya ES, Bobrovnikov SY, Kalegaev VV. 2003. Modelling of the electromagnetic field in the interplanetary space and in the Earth's magnetosphere. Space Sci Rev 107: 7-26.

Baker DN, McPherron RL, Cayton TE, Kebesadel RW. 1990. Linear prediction filter analysis of relativistic electron properties at 6.6 RE. J Geophys Res 95(A9): 15133-15140.

Baker DN, Daly E, Daglis I, Kappenman JG, Panasyuk M. 2004. Effects of space weather on technology infrastructure. Space Weather 2(2): DOI: 10.1029/2003SW000044.

Burton RK, McPherron RL, Russell CT. 1975. An empirical relationship between interplanetary conditions and Dst. J Geophys Res 80: 4204-4214.

Brueckner GE, Howard RA, Koomen MJ, Korendyke CM, Michels DJ, et al. 1995. The Large Angle Spectroscopic Coronagraph (LASCO). Sol Phys 162: 357-402. DOI: 10.1007/BF00733434.

Cheng CZF, Kuo YH, Anthes RA, Wu L. 2006. Satellite constellation monitors global and space weather. EOS Trans Am Geophys Union 87(17): 166-166.

Dolenko SA, Orlov YV, Persiantsev IG, Shugai JS. 2005. Neural network algorithm for events forecasting and its application to space physics data. Lect Notes Comput Sci 3697: 527-532.
Ganushkina NY, Liemohn MW, Kubyshkina MV, Ilie R, Singer HJ. 2010. Distortions of the magnetic field by storm time current systems in Earth's magnetosphere. Ann Geophys 28: 123-140. DOI: 10.5194/angeo-28-123-2010.

Granja C, Polansky S, Vykydal Z, Pospisil S, Owens A, Kozacek Z, Simcak M. 2016. The SATRAM Timepix spacecraft payload in open space on board the Proba-V satellite for wide range radiation monitoring in LEO orbit. Planet Space Sci 125: 114-129.

Iucci N, Levitin AE, Belov AV, Eroshenko EA, Ptitsyna NG, et al. 2005. Space weather conditions and spacecraft anomalies in different orbits. Space Weather 3(1): S01001.

Kalegaev VV. 2011. Dynamic geomagnetic field models. Geomagn Aeron 51(7): 855-865.

Kalegaev VV, Makarenkov EV. 2008. Storm-time ring and tail current dynamics under extremely disturbed conditions. J Atm Solar-Terr Phys 70: 519-525. DOI: 10.1016/j.jastp.2007.08.029.

Koons HC, Gorney DJ. 1990. A neural network model of the relativistic electron flux at geosynchronous orbit. J Geophys Res 96: 5549-5556.

Kraft S, Lupi A, Luntama JP. 2019. ESA's distributed space weather sensor system (D3S) utilizing hosted payloads for operational space weather monitoring. Acta Astronaut 156: 157-161.

Kuznetsov SN, Suvorova AV. 1998. Solar wind magnetic field and plasma during magnetopause crossings at geosynchronous orbit. Adv Space Res 22(1): 63-66.

Lanzerotti LJ, Thomson DJ, Maclennan CG. 1999. Engineering issues in space weather. In: Modern radio science, Stuchly MA (Ed.), Wiley-IEEE Press, Hoboken, NJ, pp. 25-51.

Lario D, Kwon R-Y, Vourlidas A, Raouafi NE, Haggerty DK, et al. 2016. Longitudinal properties of a Widespread Solar Energetic Particle Event on 2014 February 25: Evolution of the Associated CME Shock. Astrophys J 819: 72. DOI: 10.3847/0004-637X/819/1/72.

Lemen JR, Title AM, Akin DJ, Boerner PF, Chou C, et al. 2012. The Atmospheric Imaging Assembly (AIA) on the Solar Dynamics Observatory (SDO). Sol Phys 275: 17-40. DOI: 10.1007/s11207011-9776-8.

Ling AG, Ginet GP, Hilmer RV, Perry KL. 2010. A neural networkbased geosynchronous relativistic electron flux forecasting model. Space Weather 8(9): S09003.

Logachev YI, Bazilevskaya GA, Vashenyuk EV, Daibog EI, Ishkov VN, et al. 2014. Catalogue of solar proton events and their sources in the 20-23 cycles of solar activity. In: "Cosmic Rays and Solar Activity”, “Cosmic rays” series, 9, 155-175.

Logachev YI, Bazilevskaya GA, Vashenyuk EV, Daibog EI, Ishkov VN, et al. 2016. CATALOG of Solar Proton Events in the 23rd Cycle of Solar Activity (1996 - 2008). Logachev YI (Ed.), Moscow. DOI: 10.2205/ESDB-SAD-P-001-RU, http://www. wdcb.ru/stp/data/SPE/Catalog_SPE_23_cycle_SA.pdf.

Myagkova IN, Dolenko SA, Efitorov AO, Shirokii VR, Sentemova NS. 2017. Prediction of relativistic electron flux in the earth's outer radiation belt at geostationary orbit by adaptive methods. Geomagn Aeron 57(1): 8-15.

Patra SE, Spencer W, Horton W, Sojka J. 2011. Study of Dst/ring current recovery times using the WINDMI model. J Geophys Res 116: A02212. DOI: $10.1029 / 2010 J A 015824$.

Paulikas GA, Blake JB. 1979. Effects of the solar wind on magnetospheric dynamics: Energetic electrons at the synchronous orbit. In: Quantitative modeling of magnetospheric processes. Geophys. Monogr. Ser., Olson WP, (Ed.), AGU, Washington, DC, 21, pp. 180-202.

Podladchikova TV, Petrukovich AA. 2012. Extended geomagnetic storm forecast ahead of available solar wind measurements. Space Weather 10: S07001. 
Pembroke A, Toffoletto F, Sazykin S, Wiltberger M, Lyon J, Merkin V, Schmitt P. 2012. Initial results from a dynamic coupled magnetosphere-ionosphere-ring current model. J Geophys Res 117: A02211. DOI: 10.1029/2011JA016979.

Pulkkinen A, Rastätter L. 2009. Minimum variance analysis-based propagation of the solar wind observations: Application to realtime global magnetohydrodynamic simulations. Space Weather 7: S12001. DOI: 10.1029/2009SW000468.

Reiss MA, Temmer M, Veronig AM, Nikolic L, Vennerstrom S, et al. 2016. Verification of high-speed solar wind stream forecasts using operational solar wind models. Space Weather 14: 495-510.

Revallo M, Valach F, Hejda P, Bochníčeket J. 2014. Modeling of CME and CIR driven geomagnetic storms by means of artificial neural networks. J Atm Solar-Terr Phys 110: 9.

Schrijver CJ, Kauristie K, Aylward AD, Denardini CM, Gibson SE, et al. 2015. Understanding space weather to shield society:
A global road map for 2015-2025 commissioned by COSPAR and ILWS. Adv Space Res 55(12): 2745-2807.

Shue JH, Song P, Russell CT, Steinberg JT, Chao JK, Zastenker G, et al. 1998. Magnetopause location under extreme solar wind conditions. J Geophys Res 103(A8): 17691-17700.

Shugay Y, Slemzin V, Veselovsky I. 2014. Magnetic field sector structure and origins of solar wind streams in 2012. J Space Weather Space Clim 4: A24. DOI: 10.1051/swsc/2014021.

Shugay YS, Veselovsky IS, Seaton DB, Berghmans D. 2011. Hierarchical approach to forecasting recurrent solar wind streams. Solar Syst Res 45: 546-556. DOI: 10.1134/S0038094611060086.

Tsyganenko NA. 2002. A model of the near magnetosphere with a dawn-dusk asymmetry, 2, Parameterization and fitting to observations. J Geophys Res 107: A8. DOI: 10.1029/2001JA000220.

Tsyganenko NA, Andreeva VA. 2015. A forecasting model of the magnetosphere driven by an optimal solar wind coupling function. J Geophys Res: Space Phys 120(10): 8401-8425.

Cite this article as: Kalegaev V, Panasyuk M, Myagkova I, Shugay Y, Vlasova N, et al. 2019. Monitoring, analysis and post-casting of the Earths particle radiation environment during February 14-March 5, 2014. J. Space Weather Space Clim. 9, A29. 